\title{
MR parkinsonism index predicts vertical supranuclear gaze palsy in patients with PSP-parkinsonism \\ OPEN
}

Aldo Quattrone, MD

Maurizio Morelli, MD

David R. Williams, PhD,

FRACP

Basilio Vescio, PhD

Gennarina Arabia, MD, MSc

Salvatore Nigro, PhD

Giuseppe Nicoletti, MD

Maria Salsone, MD

Fabiana Novellino, MD

Rita Nisticò, MD

Franco Pucci, PhD

Carmelina Chiriaco, PhD

Pierfrancesco Pugliese,

MD

Domenico Bosco, MD

Manuela Caracciolo, MD

Correspondence to

Prof. Quattrone:

aldo.quattrone@gmail.com

\section{ABSTRACT}

Objective: To identify a biomarker for predicting the appearance of vertical supranuclear gaze palsy (VSGP) in patients affected by progressive supranuclear palsy-parkinsonism (PSP-P).

Methods: Twenty-four patients with PSP-P were enrolled in the current study. Patients were clinically followed up every 6 months until the appearance of VSGP or the end of the follow-up (4 years). Participants underwent MRI at baseline and at the end of follow-up. Magnetic resonance parkinsonism index (MRPI), an imaging measure useful for diagnosing PSP, was calculated.

Results: Twenty-one patients with PSP-P completed follow-up, and 3 patients dropped out. Eleven of 21 patients with PSP-P developed VSGP after a mean follow-up period of 28.5 months (range 6-48 months), while the remaining 10 patients with PSP-P did not develop VSGP during the 4-year follow-up period. At baseline, patients with PSP-P who later developed VSGP had MRPI values significantly higher than those of patients not developing VSGP without overlapping values between the 2 groups. MRPI showed a higher accuracy (100\%) in predicting VSGP than vertical ocular slowness (accuracy 33.3\%) or postural instability with or without vertical ocular slowness (accuracy $71.4 \%$ and $42.9 \%$, respectively).

Conclusions: Our study demonstrates that MRPI accurately predicted, on an individual basis, the appearance of VSGP in patients with PSP-P, thus confirming clinical diagnosis in vivo. Neurology ${ }^{\circledR}$ 2016;87:1266-1273

\section{GLOSSARY}

$\mathbf{H}-\mathbf{Y}=$ Hoehn-Yahr; $\mathbf{M C P}=$ middle cerebellar peduncle; MMSE $=$ Mini-Mental State Examination; $\mathbf{M R P I}=$ magnetic resonance parkinsonism index; PD = Parkinson disease; PSP = progressive supranuclear palsy; PSP-P = progressive supranuclear palsy-parkinsonism; RS = Richardson syndrome; $\mathbf{S C P}=$ superior cerebellar peduncle; UPDRS-ME $=$ Unified Parkinson's Disease Rating Scale-Motor Examination; VSGP = vertical supranuclear gaze palsy.

There is emerging evidence that progressive supranuclear palsy (PSP) includes 2 main clinical phenotypes: the classic Richardson syndrome (RS) and the PSP-parkinsonism (PSP-P). ${ }^{1-10} \mathrm{RS}$ is characterized by postural instability with falls and vertical supranuclear gaze palsy (VSGP) that occur within 2 years from disease onset. ${ }^{1,4,7-10}$ By contrast, PSP-P is characterized mainly by parkinsonism, resting tremor, and a moderate levodopa response with falls and VSGP that may occur later in the course of the disease..$^{1,4,7,8}$ A recent retrospective study in 19 patients with definite PSP-P demonstrated that VSGP appeared from 7 to 19 years (mean 10.8 years) after disease onset. ${ }^{6}$ Evidence indicates that VSGP is one of the more specific neurologic signs for differentiating patients with PSP-P from those with Parkinson disease (PD) ${ }^{8}$ For these reasons, in the absence of VSGP, diagnosing PSP-P may be challenging.

At present, no clinical or radiologic biomarker can predict the appearance of VSGP in patients with PSP-P. Magnetic resonance parkinsonism index (MRPI) has been proven to accurately differentiate patients with PSP from those with PD on an individual basis. ${ }^{11-19}$

From the Institute of Neurology (A.Q., M.M., G.A., F.P.), Magna Graecia University, Catanzaro, Italy; Neuroimaging Research Unit, Institute of Molecular Bioimaging and Physiology (A.Q., B.V., S.N., G.N., M.S., F.N., R.N., C.C., M.C.), National Research Council, Catanzaro, Italy; Department of Medicine (Neuroscience) (D.R.W.), Monash University, Melbourne, Australia; Neurology Unit (P.P.), Annunziata Hospital, Cosenza, Italy; and Department of Neuroscience (D.B.), San Giovanni di Dio Hospital, Crotone, Italy.

Go to Neurology.org for full disclosures. Funding information and disclosures deemed relevant by the authors, if any, are provided at the end of the article. The Article Processing Charge was paid by the authors.

This is an open access article distributed under the terms of the Creative Commons Attribution-NonCommercial-NoDerivatives License 4.0 (CC BY-NC-ND), which permits downloading and sharing the work provided it is properly cited. The work cannot be changed in any way or used commercially. 
Moreover, the MRPI can predict the evolution of undetermined parkinsonisms toward PSP phenotypes, thus suggesting its usefulness for identifying patients with PSP in very early stages of the disease. ${ }^{20,21}$ In the current study, we investigated the possible usefulness of MRPI in predicting the appearance of VSGP in patients with PSP-P.

METHODS Patients. Twenty-four patients with a clinical diagnosis of PSP-P were consecutively recruited from among those referred to the Institute of Neurology, University Magna Graecia, Catanzaro, Italy, between May 2009 and September 2014. One of the authors (M.M.) with $>10$ years of experience in movement disorders clinically evaluated all patients. We made the diagnosis of PSP-P according to internationally accepted clinical criteria and expert guidelines. ${ }^{1,4}$ Diagnostic criteria for PSP-P included asymmetric bradykinesia or tremor associated with at least one of the following clinical features developed after 2 years from disease onset: isolated postural instability with backward falls, isolated slowness of vertical saccades, or both postural instability with backward falls and slowness of vertical saccades. ${ }^{1,4}$ For each patient, a complete medical history, neurologic examination, and clinical assessment with the Unified Parkinson's Disease Rating Scale-Motor Examination (UPDRS-ME) ${ }^{22}$ and Hoehn-Yahr (H-Y) rating scale $^{23}$ (off medications overnight) were available. We used the Mini-Mental State Examination (MMSE) ${ }^{24}$ to assess cognitive performance in all patients. Exclusion criteria were history of neuroleptic use within the past 6 months, presence of serum or urinary abnormalities (iron, ferritin, transferrin, calcium, parathormone, ceruloplasmin), evidence of normal striatal uptake in dopamine transporter ${ }^{123}$ I-FP-CIT-SPECT, or evidence on the MRI scan of vascular lesions in the basal ganglia.

Standard protocol approvals, registrations, and patient consents. All study procedures and ethics aspects were approved by the institutional review board. In addition, written informed consent was obtained from all participants who were examined as part of the study.

MRI acquisition and analysis. All patients had brain MRI with a 3T MR750 GE MRI scanner and an 8-channel head coil. All study participants underwent the same MRI protocol, including 3-dimensional T1-weighted volumetric spoiled gradient echo (sagittal section; repetition time/echo time 9.2/ 3.7 milliseconds; slice thickness $1.0 \mathrm{~mm}$; frequency and phaseencoding matrix $256 \times 256$; flip angle $12^{\circ}$; field of view $25.6 \mathrm{~mm}$ ), T2-weighted fast spin echo (axial section; repetition time/echo time 5462/85 milliseconds; slice thickness $4.0 \mathrm{~mm}$; frequency and phase-encoding matrix $512 \times 256$; field of view $24 \mathrm{~mm}$ ), and T2-weighted fluid-attenuated inversion recovery (axial section; repetition time/echo time/inversion time 9500/ 100/2250 milliseconds; slice thickness $4.0 \mathrm{~mm}$; frequency and phase-encoding matrix $512 \times 256)$ sequences. Two independent raters with $>10$ years of experience in neuroradiology who were blinded to patient diagnoses analyzed the images. Midbrain area, pons area, middle cerebellar peduncle (MCP) width, and superior cerebellar peduncle (SCP) width were measured according to the methods described previously. ${ }^{11,25}$ The MRPI was calculated by multiplying the midsagittal area of the pons/midsagittal area of midbrain $(\mathrm{P} / \mathrm{M})$ by the MCP width/SCP width: $[(\mathrm{P} / \mathrm{M}) \times$ (MCP/SCP)]. ${ }^{11}$

Study design. In this cohort study, patients underwent neurologic examination and MRI at baseline and at the end of the follow-up. At baseline and follow-up, levodopa response was assessed both in the off state (off medication overnight) and 2 hours after drug administration as a clinical improvement of $\geq 20 \%$ on the UPDRS-ME score. Patients were clinically followed up every 6 months by one of the authors (M.M.) who was blinded to the MRI measurements. For each patient with PSP-P, follow-up was calculated as the time interval between the date of baseline evaluation (clinical examination and MRI measurements) and the appearance of clinical evidence of VSGP with normal vestibular-ocular reflex or as the time period of 4 years in the absence of VSGP. Palsy of both voluntary downward and upward vertical gaze and palsy of downward and marked limitation of upward vertical gaze were the established ocular motor criteria for the diagnosis of VSGP. ${ }^{26}$

Intrarater and interrater evaluation. To assess the intrarater reliability, each measurement was repeated (images in a different order) 2 weeks later by the same rater who was blinded to the first MRI evaluation. Two independent raters performed interrater evaluation in both MRI evaluations (baseline and follow-up).

Statistical analysis. The differences at baseline evaluation in sex distribution, levodopa responsiveness, and clinical features between patients with PSP-P who developed VSGP at followup and those who did not were assessed with the Fisher exact test. At follow-up, the Fisher exact test was also used to compare levodopa responsiveness between patients with PSP-P developing VSGP and those not developing VSGP. The Shapiro-Wilk test was used to assess data distribution properties to choose the most appropriate statistical test for group comparisons. At baseline evaluation, to assess differences between patients with PSP-P developing VSGP and those not developing VSGP, the $t$ test was used to compare age at examination, age at disease onset, disease duration, pons area, midbrain area, MCP width, and SCP width, while differences in MMSE score, UPDRS-ME score, H-Y score, and MRPI were assessed by means of the Mann-Whitney $U$ test. At follow-up, the $t$ test was also used to compare age at examination, disease duration, pons area, midbrain area, SCP width, and MRPI, while the Mann-Whitney test was used to compare MMSE score, UPDRS-ME score, H-Y score, and MCP width. Moreover, we calculated the optimal cutoff values for midbrain area, SCP width, and MRPI as the levels with the maximum sum of sensitivity and specificity using the receiveroperating characteristic curve analysis. Sensitivity, specificity, positive predictive value, negative predictive value, and diagnostic accuracy were determined for differentiating patients with PSP-P developing VSGP from those not developing VSGP using clinical features and brain MRI measurements. The McNemar test was used to compare the diagnostic accuracy of clinical features and MRPI in differentiating patients with PSP-P developing VSGP from those not developing VSGP. To assess differences in MRPI value between baseline and the follow-up evaluation, a mixed-model analysis of variance was performed, considering status (developing, not developing) as a between factor and time (baseline, follow-up) as a within factor. To assess the intrarater and interrater reliabilities, the intraclass correlation coefficient was calculated. All tests were 2 tailed, and the $\alpha$ level was set at $p<0.05$. Statistical analysis was performed with $\mathrm{R}$ statistical software ( $\mathrm{R}$ for Unix/Linux, 
version 2.15.1, R Foundation for Statistical Computing, Vienna, Austria, 2012).

RESULTS Demographic, clinical, and radiologic data of patients with PSP-P are listed in table 1. Twenty-one of 24 patients with PSP-P completed the study; 3 patients (1 patient with slowness of vertical saccades and 2 patients with postural instability with backward falls and slowness of vertical saccades) dropped out. In particular, 1 patient died and 2 patients were lost during the follow-up.

Eleven of 21 patients (52.3\%) with PSP-P developed VSGP during the follow-up. The mean \pm SD duration of clinical follow-up period for these patients was $28.5 \pm 13.6$ months (range 6-48 months). None of the remaining 10 patients with PSP-P developed VSGP within 48 months of the clinical observational period. Baseline comparisons of clinicoradiologic data between patients with PSP-P developing and not developing VSGP are shown in table 1 and figure 1 . More than $50 \%$ of patients in both groups showed levodopa responsiveness. It is noteworthy that the patients with PSP-P who developed VSGP showed baseline MRPI values (mean 14.77; range 12.52-20.24) that were significantly higher $(p<0.001)$ than those detected in patients who did not develop VSGP (mean 9.77; range 7.90-11.34) without overlapping values between the 2 groups (table 1 ). Follow-up comparisons of clinicoradiologic data between these 2 patient groups are shown in table 2 . Of note, most patients in both groups lost levodopa responsiveness, with this result being more evident in those patients who developed VSGP. Comparisons between MRPI values in patients with PSP-P

\begin{tabular}{|c|c|c|c|c|c|}
\hline \multirow[t]{2}{*}{ Table 1} & \multicolumn{5}{|c|}{$\begin{array}{l}\text { Baseline clinicoradiologic data in the whole group of patients with progressive supranuclear palsy-parkinsonism (PSP-P) and in } \\
\text { patients developing and not developing vertical supranuclear gaze palsy (VSGP) }\end{array}$} \\
\hline & & PSP-P (whole sample) & PSP-P developing VSGP & PSP-P not developing VSGP & $p$ Value $^{a}$ \\
\hline$M / F, n$ & & $19 / 5$ & $10 / 1$ & $6 / 4$ & $0.15^{c}$ \\
\hline Age at ex & ination, $y^{d}$ & $68.5 \pm 6.1(57-78)$ & $68.9 \pm 4.9(58-75)$ & $68.7 \pm 7.7(57-78)$ & $0.94^{\mathrm{e}}$ \\
\hline Disease $c$ & tion, $y^{d}$ & $5.9 \pm 2.2(3-9)$ & $6.3 \pm 2.4(3-9)$ & $5.6 \pm 2.2(3-9)$ & $0.51^{e}$ \\
\hline MMSE sc & & $24(17-29)$ & $23.5(17-25)$ & $24.5(22-27)$ & $0.21^{\mathrm{g}}$ \\
\hline UPDRS-N & $\operatorname{core}^{f}$ & $31.5(16-48)$ & $34(31-42)$ & $27(16-37)$ & $0.001^{9}$ \\
\hline $\mathrm{H}-\mathrm{Y}$ score & & $3.0(2-5)$ & $3(3-5)$ & $2(2-3)$ & $<0.001^{9}$ \\
\hline $\begin{array}{l}\text { Slowne } \\
\text { of disea }\end{array}$ & $\begin{array}{l}\text { of vertical saccades after } 2 \text { y } \\
\text { onset }\end{array}$ & 14 & 5 & 8 & $0.18^{c}$ \\
\hline $\begin{array}{l}\text { Postur: } \\
\text { slowne: } \\
\text { disease }\end{array}$ & $\begin{array}{l}\text { stability with backward falls and } \\
\text { f vertical saccades after } 2 \text { y of } \\
\text { set }\end{array}$ & 9 & 6 & 1 & $0.063^{\mathrm{c}}$ \\
\hline \multicolumn{6}{|c|}{ Brain MRI measurements ${ }^{d}$} \\
\hline Pons ar & $\mathrm{mm}^{2}$ & $476.0 \pm 57.1(380-585)$ & $478.5 \pm 58.1(398-562)$ & $478.1 \pm 58.1(407-585)$ & $0.99^{e}$ \\
\hline Midbrai & rea, $\mathrm{mm}^{2}$ & $98.6 \pm 20.2(58-136)$ & $86.7 \pm 14.2(58-110)$ & $110.7 \pm 13.8(88-136)$ & $0.001^{\mathrm{e}}$ \\
\hline MCP wi & $\mathrm{mm}$ & $8.5 \pm 0.8(6.3-10.0)$ & $8.5 \pm 1.0(6.3-10.0)$ & $8.6 \pm 0.6(8.0-9.7)$ & $0.60^{\mathrm{e}}$ \\
\hline
\end{tabular}

Abbreviations: $\mathrm{H}-\mathrm{Y}=$ Hoehn-Yahr; $\mathrm{MCP}=$ middle cerebellar peduncles; MMSE = Mini-Mental State Examination; MRPI = magnetic resonance parkinsonism index; SCP = superior cerebellar peduncles; UPDRS-ME = Unified Parkinson's Disease Rating Scale-Motor Examination.

${ }^{a}$ Clinicoradiologic comparisons between patients with PSP-P developing and not developing VSGP.

${ }^{b}$ Three of 24 patients with PSP-P were lost during follow-up.

${ }^{\mathrm{c}}$ Fisher exact test.

${ }^{\mathrm{d}}$ Data are expressed as mean \pm SD (range).

e The $t$ test.

${ }^{\mathrm{f}}$ Data are expressed as median (range).

${ }^{g}$ Mann-Whitney U test.

${ }^{\mathrm{h}}$ Number (percentage) of patients who showed a clinical improvement of $\geq 20 \%$ in comparison with that detected in the off state. 
Figure $1 \quad$ MRI measurements in patients with progressive supranuclear palsyparkinsonism (PSP-P) developing or not developing vertical supranuclear gaze palsy (VSGP)
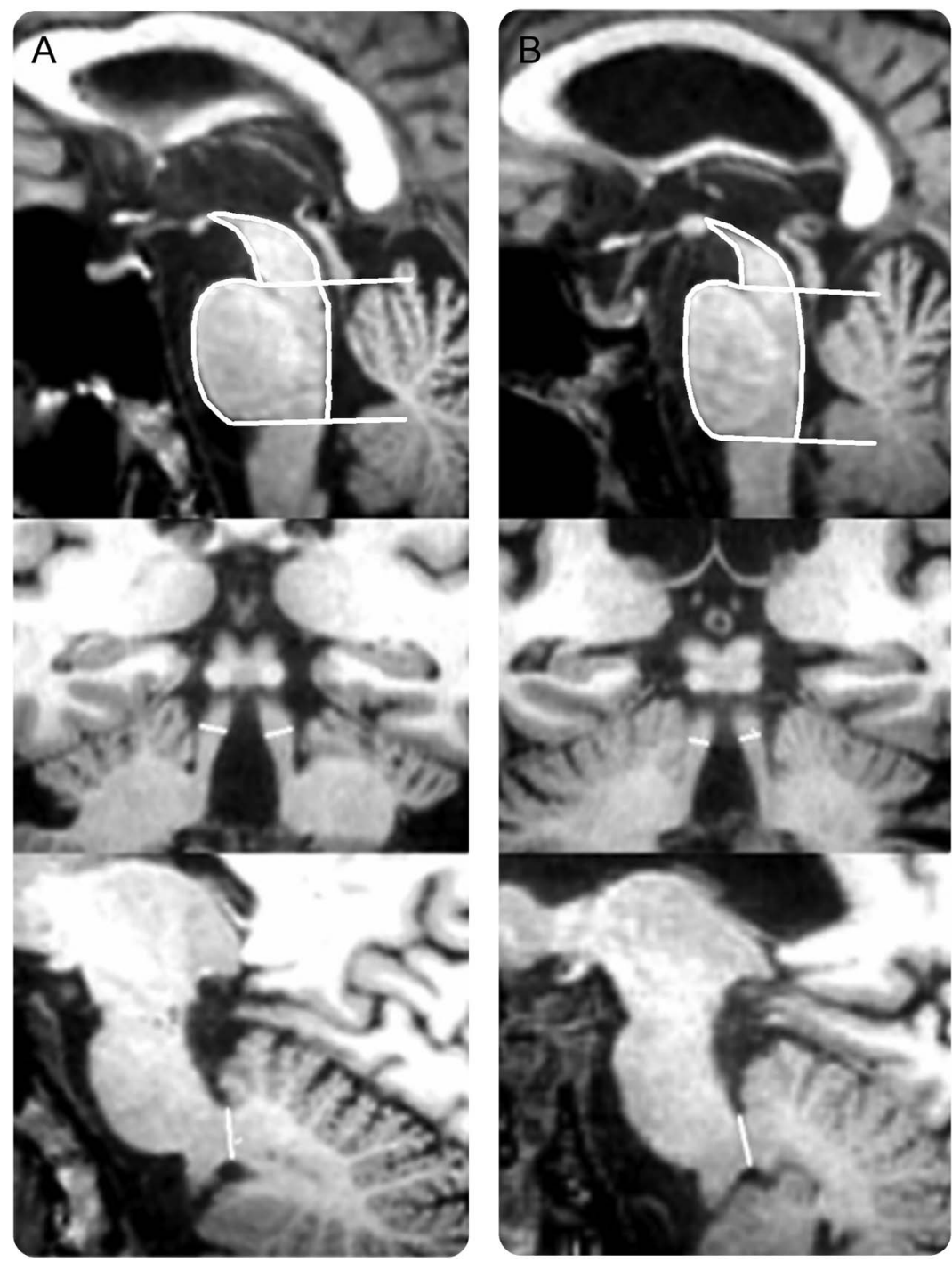

Midsagittal T1-weighted volumetric spoiled gradient echo (GE) MRIs of midbrain and pons areas (top row). Coronal T1-weighted volumetric spoiled GE MRIs of superior cerebellar peduncle (SCP) width (intermediate row). Midsagittal T1-weighted volumetric spoiled GE MRIs of middle cerebellar peduncle (MCP) width (bottom row). (A) A patient with PSP-P not developing VSGP; (B) a patient with PSP-P developing VSGP. (A) Midbrain area $108 \mathrm{~mm}^{2}$; pons area $422 \mathrm{~mm}^{2}$; SCP width $3.65 \mathrm{~mm}$; MCP width $8.95 \mathrm{~mm}$; magnetic resonance parkinsonism index (MRPI) 9.58. (B) Midbrain area $88 \mathrm{~mm}^{2}$; pons area $434 \mathrm{~mm}^{2}$; SCP width $2.7 \mathrm{~mm}$; MCP width $7.8 \mathrm{~mm}$; MRPI 14.25 .

developing and not developing VSGP at baseline and follow-up are shown in figure 2. The mixedmodel analysis of variance showed a significant effect of status (developing, not developing; $p<$ 0.001 ) and no significant effect of time (baseline, follow-up; $p=0.38$ ), while the interaction of status and time was shown to be slightly significant $(p=0.045)$, thus suggesting that the change in MRPI values from baseline to follow-up evaluation was higher in patients with PSP-P developing VSGP than in those not developing VSGP.
Sensitivity, specificity, positive predictive value, negative predictive value, and diagnostic accuracy of baseline clinical features and MRI measurements to predict VSGP in patients with PSP-P are summarized in table 3.

There was an excellent intrarater correlation between repeated MRI measurements at baseline (intraclass correlation coefficient: pons area 0.991; midbrain area 0.992 ; MCP width 0.988 ; SCP width 0.987 ) and at follow-up (intraclass correlation coefficient: pons area 0.989 ; midbrain area 0.991; MCP width 0.992; SCP width 0.990). Moreover, there was an excellent interrater correlation in both measurements at baseline (intraclass correlation coefficient: pons area 0.990 ; midbrain area 0.987 ; MCP width 0.986; SCP width 0.988 ) and follow-up (intraclass correlation coefficient: pons area 0.986 ; midbrain area 0.993 ; MCP width 0.991; SCP width 0.989).

DISCUSSION The results of our study show that MRPI is a highly accurate magnetic resonance measure for predicting VSGP in patients with clinical phenotypes that fulfilled the criteria for PSP-P. In particular, MRPI is more powerful in predicting VSGP than clinical features suggestive of PSP-P such as slowness of vertical saccades and postural instability with or without slowness of vertical saccades.

In the last decade, a new nosology for $\mathrm{PS}^{1-9}$ has emerged that confirmed the original observations ${ }^{10}$ but also separated out several clinical subtypes that would otherwise not satisfy the established consensus criteria for PSP. ${ }^{26}$ According to the results of a large clinicopathologic series of patients with PSP, some researchers renamed the classic PSP form as RS and proposed the term PSP-P for the clinical phenotype similar to PD, which accounted for up to one-third of their PSP cases. ${ }^{1,4}$ The major difference relates to the regional distribution of pathology and the resulting clinical phenomenology with greater tau burden in RS than in PSP-P. ${ }^{1-6}$ In the early stage of the disease, PSP-P phenotype shows a clinical picture similar to that observed in patients with PD characterized by asymmetrical onset, resting tremor, rigidity, moderate initial response to levodopa, a later age at onset, and a more favorable disease course compared with RS. ${ }^{1,4}$ Indeed, in a recent retrospective review, ${ }^{8}$ no clinical feature distinguished PSP-P from PD in the early stage of the disease. VSGP appeared to be the most specific clinical sign of PSP-P compared to PD and multiple system atrophy, ${ }^{8}$ but this clinical sign often occurs later during the course of the disease. A recent retrospective study in 19 patients with definite PSP-P demonstrated that VSGP appeared from 7 to 19 years (mean 10.8 years) after disease onset. ${ }^{6}$ Thus, at the 


\begin{tabular}{|c|c|c|c|c|}
\hline \multirow[t]{2}{*}{ Table 2} & \multicolumn{4}{|c|}{$\begin{array}{l}\text { Clinicoradiologic comparisons in patients with progressive supranuclear palsy-parkinsonism (PSP-P) } \\
\text { developing and not developing vertical supranuclear gaze palsy (VSGP) at the end of follow-up }\end{array}$} \\
\hline & & PSP-P developing VSGP & PSP-P not developing VSGP & $p$ Value \\
\hline $\mathrm{n}$ & & 11 & 10 & - \\
\hline$M / F, n$ & & $10 / 1$ & $6 / 4$ & $0.15^{\mathrm{a}}$ \\
\hline Age at ex & ination, $y^{b}$ & $71.4 \pm 5.4$ (60-79) & $73.0 \pm 7.5(61-82)$ & $0.58^{\mathrm{c}}$ \\
\hline Disease $c$ & tion, $y^{b}$ & $9.3 \pm 3.4(5-15)$ & $10.0 \pm 2.5(7-14)$ & $0.58^{c}$ \\
\hline MMSE sc & & 20.5 (19-21) & $20.0(19-24)$ & $0.94^{e}$ \\
\hline UPDRS-M & score $^{d}$ & $41(36-46)$ & 32 (21-39) & $0.001^{e}$ \\
\hline $\mathrm{H}-\mathrm{Y}$ score & & $4(3-5)$ & $2.5(2-3)$ & $0.001^{\mathrm{e}}$ \\
\hline Levodopa & sponsiveness $^{f}$ & 2 (18.1) & $3(30)$ & $0.64^{\mathrm{a}}$ \\
\hline \multicolumn{5}{|c|}{ Brain MRI measurements ${ }^{\mathrm{b}, \mathrm{g}}$} \\
\hline \multicolumn{2}{|c|}{ Pons area, $\mathrm{mm}^{2}$} & $480.7 \pm 60.4(375-545)$ & $440.4 \pm 41.8(378-502)$ & $0.14^{c}$ \\
\hline \multicolumn{2}{|c|}{ Midbrain area, $\mathrm{mm}^{2}$} & $82.4 \pm 11.1(65-96)$ & $111.0 \pm 11.2(99-133)$ & $<0.001^{\mathrm{c}}$ \\
\hline \multicolumn{2}{|c|}{ MCP width, mm } & $8.6 \pm 0.8(7.1-9.9)$ & $8.7 \pm 0.7(8.1-9.8)$ & $0.87^{e}$ \\
\hline \multicolumn{2}{|c|}{ SCP width, mm } & $3.26 \pm 0.5(2.50-3.85)$ & $3.60 \pm 0.3(3.20-4.20)$ & $0.14^{\mathrm{c}}$ \\
\hline \multicolumn{2}{|l|}{ MRPI } & $15.48 \pm 1.3(14.11-17.86)$ & $9.76 \pm 1.6(7.37-11.57)$ & $<0.001^{\mathrm{c}}$ \\
\hline
\end{tabular}

Abbreviations: $\mathrm{H}-\mathrm{Y}=$ Hoehn-Yahr; $\mathrm{MCP}=$ middle cerebellar peduncles; MMSE = Mini-Mental State Examination; MRPI = magnetic resonance parkinsonism index; SCP $=$ superior cerebellar peduncles; UPDRS-ME = Unified Parkinson's Disease Rating Scale-Motor Examination.

${ }^{a}$ Fisher exact test.

${ }^{b}$ Data are expressed as mean \pm SD (range).

${ }^{\mathrm{c}}$ The $t$ test.

${ }^{\mathrm{d}}$ Data are expressed as median (range).

${ }^{\mathrm{e}}$ Mann-Whitney $U$ test.

${ }^{f}$ Number (percentage) of patients who showed a clinical improvement of $\geq 20 \%$ in comparison with that detected in the off state.

${ }^{9}$ Nine of 11 patients (81.8\%) with PSP-P developing VSGP and 7 of 10 patients (70\%) with PSP-P not developing VSGP repeated the MRI examination at the end of follow-up period.

present time, diagnosing PSP-P may be challenging in the early phase of the disease in the absence of VSGP. To the best of our knowledge, no biomarker exists
Box plots of baseline and follow-up magnetic resonance parkinsonism index measurements in patients with progressive supranuclear palsyparkinsonism (PSP-P) developing (A) or not developing (B) vertical supranuclear gaze palsy (VSGP)
A. PSP-P developing VSGP

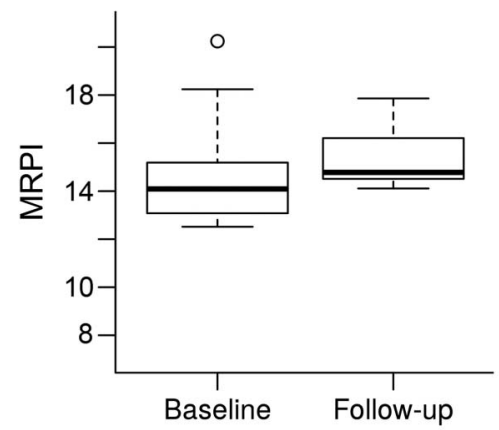

B. PSP-P not developing VSGP

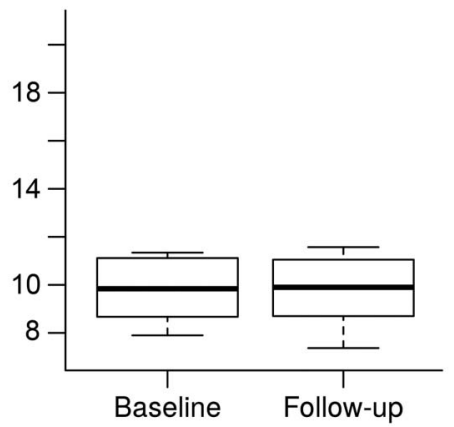

Box plots show an increase of MRPI value in patients with PSP-P developing VSGP in comparison with those not developing VSGP (mixed-model analysis of variance, $p=0.045$ ). Vertical solid lines (whiskers) show lower and upper values. Box stretches from lower hinge (25th percentile) to upper hinge (75th percentile). Median is shown as a line across each box. that is capable of predicting the appearance of VSGP in patients with clinical features fulfilling criteria for PSP-P.

In the last few years, several MRI measurements such as the ratio of midbrain to pons areas ${ }^{27-32}$ and $\mathrm{MRPI}^{11-19}$ have been reported to accurately diagnose patients with PSP. Both MRI measurements have proven to be useful tools for diagnosing PSP, ${ }^{11-19,27-32}$ but the ratio of midbrain to pons areas was reported to be influenced by aging ${ }^{33}$ and thus is less reliable than MRPI for diagnosing this disease, which typically occurs in older people. Moreover, increased MRPI values were reported to be capable of predicting PSP in patients with undetermined parkinsonisms, a finding of great interest for diagnosing these diseases at a very early stage. ${ }^{21}$

In the current study, we found that 11 of $21 \mathrm{pa}-$ tients with PSP-P developed VSGP after a mean follow-up period of 28.5 months (range 6-48 months), while the remaining 10 patients with PSP-P did not develop VSGP during the follow-up period of up to 4 years. These 2 groups did not show significant differences in baseline demographic or clinical variables except for UPDRS-ME and H-Y scores, which were 


\begin{tabular}{|c|c|c|c|c|c|c|}
\hline \multirow[t]{2}{*}{ Table 3} & \multicolumn{6}{|c|}{$\begin{array}{l}\text { Performances of clinical features and magnetic resonance parkinsonism index (MRPI) values for } \\
\text { predicting vertical supranuclear gaze palsy in patients with progressive supranuclear palsy- } \\
\text { parkinsonism }\end{array}$} \\
\hline & & Sensitivity, \% & Specificity, \% & PPV, \% & NPV, \% & Accuracy, \% \\
\hline \multicolumn{7}{|c|}{ Clinical features } \\
\hline \multicolumn{2}{|c|}{$\begin{array}{l}\text { Isolated postural instability with backward } \\
\text { falls after } 2 \text { y of disease onset }\end{array}$} & 0 & 90 & 0 & 45 & 42.9 \\
\hline \multicolumn{2}{|c|}{$\begin{array}{l}\text { Slowness of vertical saccades after } 2 \text { y of } \\
\text { disease onset }\end{array}$} & 45.5 & 20.0 & 38.5 & 25.0 & 33.3 \\
\hline \multicolumn{2}{|c|}{$\begin{array}{l}\text { Postural instability with backward falls and } \\
\text { slowness of vertical saccades after } 2 \text { y of } \\
\text { disease onset }\end{array}$} & 54.6 & 90.0 & 85.7 & 64.3 & 71.4 \\
\hline \multicolumn{7}{|c|}{ MRI measurements } \\
\hline \multicolumn{2}{|c|}{ Midbrain area (cutoff value $\leq 95.0)^{a}$} & 81.8 & 90 & 90 & 81.8 & 85.7 \\
\hline \multicolumn{2}{|c|}{ SCP width (cutoff value $\leq 3.41)^{\mathrm{a}}$} & 63.6 & 100 & 100 & 71.4 & 81.0 \\
\hline \multicolumn{2}{|c|}{ MRPI (cutoff value $\geq 12.52)^{a}$} & 100 & 100 & 100 & 100 & 100 \\
\hline
\end{tabular}

Abbreviations: NPV = negative predictive value; PPV = positive predictive value; $\mathrm{SCP}=$ superior cerebellar peduncles. McNemar tests showed significant differences in comparing the diagnostic accuracies of MRPI vs isolated postural instability with backward falls after the second year of disease onset $(p=0.001)$, MRPI vs slowness of vertical saccades after 2 years of disease onset $(p<0.001)$, and MRPI vs postural instability with backward falls after the second year of disease onset and slowness of vertical saccades $(p=0.04)$.

${ }^{a}$ Cutoff values were determined as the values with the highest sum of sensitivity and specificity.

slightly higher in patients developing VSGP with a large overlap between the 2 groups. It is noteworthy that patients with PSP-P who developed VSGP showed at baseline significantly higher MRPI values (mean 14.77; range 12.52-20.24) than those detected in patients with PSP-P who did not develop VSGP at the end of follow-up (mean 9.77; range 7.90-11.34) without overlapping values between the 2 groups. This result is in accordance with a previous study showing that among patients with undetermined parkinsonism, baseline MRPI had higher values in patients who later developed PSP compared with those not developing this disease. At the end of the follow-up period, the MRPI values of patients who developed VSGP were significantly higher than those detected at baseline (mean 15.48; range 14.1117.86), whereas no differences between baseline and follow-up MRPI values (mean 9.76; range 7.3711.57) were observed in patients not developing VSGP (figure 2). Our findings therefore indicate that patients with PSP-P who developed VSGP had, at the end of follow-up, a worsening atrophy of infratentorial brain structures measured by MRPI, a finding not observed in patients with PSP-P not developing VSGP.

Of note, the patients with PSP-P not developing VSGP showed baseline MRPI values very similar to those reported in our recent study ${ }^{33}$ in patients with PD with similar motor disability scores (MRPI mean values 9.77 vs 9.85 , respectively). Although baseline MRPI was not different between these 2 groups, the PSP-P midbrain area value was smaller than that detected in PD, suggesting that this brain structure may be involved early in patients with PSP-P not developing VSGP. Why patients with PSP-P not developing VSGP had baseline and follow-up MRPI values lower than those developing VSGP is still unknown. Because the clinical development of VSGP in patients with PSP-P may take up to 17 years, ${ }^{6}$ it is possible that the patients not developing VSGP after 4 years of follow-up may require a longer period of clinical observation before developing VSGP.

Alternatively, because falls occur very frequently in the elderly and ocular slowness is a clinically difficult sign to evaluate, it is possible that some patients with PD have been misdiagnosed as having PSP-P. However, because the large majority of these patients not developing VSGP (7 of 10) showed no levodopa responsiveness at the end of follow-up, the diagnosis of PD seems unlikely. Whether these patients can be considered to have PSP-P in a very early stage or are affected by other forms of parkinsonism needs to be elucidated. Pathologic studies in these patients not developing VSGP are warranted.

Of note, in patients with PSP-P developing VSGP, baseline MRPI showed a higher sensitivity $(100 \%)$ and specificity $(100 \%)$ in predicting the clinical development of VSGP than clinical features typically occurring in PSP-P. The combination of postural instability with backward falls associated with slowness of vertical saccades showed an accuracy of $71.4 \%$, while isolated postural instability with backward falls or isolated slowness of vertical saccades had an accuracy of $42.9 \%$ and $33.3 \%$, respectively. These results are in agreement with those of our previous study ${ }^{21}$ in which we demonstrated that clinical 
features suggestive of PSP such as slowness of vertical saccades or first-year falls had a lower accuracy (61.9\% and $73.8 \%$, respectively) than MRPI $(92.9 \%)$ in predicting the evolution of undefined parkinsonisms toward clinical phenotypes that fulfilled the diagnostic criteria for possible or probable PSP. ${ }^{26}$

There were some limitations to this study. We used clinical criteria for the diagnoses of disease, and none of our patients underwent autopsy. Thus, it is possible that in some patients, including those with PSP-P with normal MRPI, the clinical diagnosis may be in error. However, these clinical evaluations had been performed according to operational diagnostic criteria and expert guidelines ${ }^{1,4,26,34}$ and were carried out by a specialist in movement disorders with extensive experience in diagnosis and management of PSP. In the current study, MRI was performed with a $3 \mathrm{~T}$ scanner, which is not always available in movement disorder clinics. However, we previously performed several studies on MRPI using a $1.5 \mathrm{~T}$ scanner, ${ }^{11,15,21}$ supporting the reliability of $1.5 \mathrm{~T}$ scanners for MRPI measurements also in patients with PSP-P. ${ }^{21}$

The small sample size of patients with PSP-P is another limitation of our study; however, PSP-P is a rare disease, and imaging data repeated over time were difficult to obtain. The large majority of previous studies of PSP-P enrolled a number of participants similar to ours. However, larger studies with longer follow-up times are needed to confirm our results.

Our study demonstrates that MRPI was more powerful than clinical features in predicting the appearance of VSGP in patients with PSP-P on an individual basis. VSPG remains one of more specific signs for diagnosing PSP-P, but its appearance in some patients may require many years after disease onset. Therefore, the negative predictive value of baseline MRPI is strictly for VSGP at 4 years of follow-up and not necessarily for the diagnosis of PSP-P. Our study showed that MRPI accurately predicts VSGP and may help distinguish PSP-P from other parkinsonisms in early stages of the disease.

\section{AUTHOR CONTRIBUTIONS}

Prof. Quattrone: drafting/revising the manuscript, study concept or design, analysis or interpretation of data, study supervision. Dr. Morelli: drafting/revising the manuscript, analysis or interpretation of data, acquisition of data. Dr. Williams: drafting/revising the manuscript, analysis or interpretation of data. Dr. Vescio: analysis or interpretation of data, statistical analysis. Dr. Arabia: drafting/revising the manuscript, analysis or interpretation of data. Dr. Nigro: analysis or interpretation of data. Dr. Nicoletti: analysis or interpretation of data. Dr. Salsone: analysis or interpretation of data. Dr. Novellino: analysis or interpretation of data. Dr. Nisticò: analysis or interpretation of data. Dr. Pucci: analysis or interpretation of data. Dr. Chiriaco: acquisition of data. Dr. Pugliese: acquisition of data. Dr. Bosco: acquisition of data. Dr. Caracciolo: acquisition of data.

\section{STUDY FUNDING}

This study was supported by the PON Neuromeasures (PON03PE_00009_1).

\section{DISCLOSURE}

The authors report no disclosures relevant to the manuscript. Go to Neurology.org for full disclosures.

Received February 16, 2016. Accepted in final form June 7, 2016.

\section{REFERENCES}

1. Williams DR, de Silva R, Paviour DC, et al. Characteristics of two distinct clinical phenotypes in pathologically proven progressive supranuclear palsy: Richardson's syndrome and PSP-parkinsonism. Brain 2005;128:12471258.

2. Williams DR, Holton JL, Strand C, et al. Pathological tau burden and distribution distinguishes progressive supranuclear palsy-parkinsonism from Richardson's syndrome. Brain 2007;130:1566-1576.

3. Dickson DW, Rademakers R, Hutton ML. Progressive supranuclear palsy: pathology and genetics. Brain Pathol 2007;17:74-82.

4. Williams DR, Lees AJ. Progressive supranuclear palsy: clinicopathological concepts and diagnostic challenges. Lancet Neurol 2009;8:270-279.

5. Lang AE. Clinical heterogeneity in progressive supranuclear palsy: challenges to diagnosis, pathogenesis and future therapies. Mov Disord 2014;29:1707-1709.

6. Respondek G, Stamelou M, Kurz C, et al. The phenotypic spectrum of progressive supranuclear palsy: a retrospective multicenter study of 100 definite cases. Mov Disord 2014; 29:1758-1766

7. Williams DR, Lees AJ, Wherrett JR, Steele JC. J. Clifford Richardson and 50 years of progressive supranuclear palsy. Neurology 2008;70:566-573.

8. Williams DR, Lees AJ. What features improve the accuracy of the clinical diagnosis of progressive supranuclear palsy-parkinsonism (PSP-P)? Mov Disord 2010;25:357-362.

9. Liscic RM, Srulijes K, Gröger A, Maetzler W, Berg D. Differentiation of progressive supranuclear palsy: clinical, imaging and laboratory tolls. Acta Neurol Scand 2013; 127:362-370.

10. Richardson JC, Steele J, Olszewski J. Supranuclear ophthalmoplegia, pseudobulbar palsy, nuchal dystonia and dementia: a clinical report on eight cases of "heterogenous system degeneration." Trans Am Neurol Assoc 1963;88: 25-29.

11. Quattrone A, Nicoletti G, Messina D, et al. MR imaging index for differentiation of progressive supranuclear palsy from Parkinson disease and the Parkinson variant of multiple system atrophy. Radiology 2008; 246:214-221.

12. Hotter A, Esterhammer R, Schocke MF, Seppi K. Potential of advanced MR imaging techniques in the differential diagnosis of parkinsonism. Mov Disord 2009;24:S711-S720.

13. Hussl A, Mahlknecht P, Scherfler C, et al. Diagnostic accuracy of the magnetic resonance parkinsonism index and the midbrain-to-pontine area ratio to differentiate progressive supranuclear palsy from Parkinson's disease and the Parkinson variant of multiple system atrophy. Mov Disord 2010;25:2444-2449.

14. Lehéricy S, Hartmann A, Lannuzel A, et al. Magnetic resonance imaging lesion pattern in Guadeloupean 
parkinsonism is distinct from progressive supranuclear palsy. Brain 2010;133:2410-2425.

15. Morelli M, Arabia G, Salsone M, et al. Accuracy of magnetic resonance parkinsonism index for differentiation of progressive supranuclear palsy from probable or possible Parkinson disease. Mov Disord 2011;26:527-533.

16. Jones N. Movement disorders: imaging differentiates progressive supranuclear palsy from Parkinson disease. Nat Rev Neurol 2011;7:186.

17. Stamelou M, Knake S, Oertel WH, Höglinger GU. Magnetic resonance imaging in progressive supranuclear palsy. J Neurol 2011;258:549-558.

18. Massey LA, Micallef C, Paviour DC, et al. Conventional magnetic resonance imaging in confirmed progressive supranuclear palsy and multiple system atrophy. Mov Disord 2012;27:1754-1762.

19. Colosimo C, Bak TH, Bologna M, Berardelli A. Fifty years of progressive supranuclear palsy. J Neurol Neurosurg Psychiatry 2014;85:938-944.

20. Karimi M, Perlmutter JS. MRI measures predict progressive supranuclear palsy: clinically useful? Neurology 2011; 77:1028-1029.

21. Morelli M, Arabia G, Novellino F, et al. MRI measurements predict PSP in unclassifiable parkinsonisms: a cohort study. Neurology 2011;77:1042-1047.

22. Fahn S, Elton RL. Unified Parkinson's Disease Rating Scale. In: Fahn S, Marsden CD, Calne D, Goldstein M, eds. Recent Developments in Parkinson's Disease. Florham Park, NJ: MacMillan Healthcare Information; 1987: 153-163.

23. Hoehn MM, Yahr MD. Parkinsonism: onset, progression, and mortality. Neurology 1967;17:427-442.

24. Folstein MF, Folstein SE, McHugh PR. "Mini-mental state:" a practical method for grading the cognitive state of patients for the clinician. J Psychiatr Res 1975;12:189-198.
25. Nicoletti G, Fera F, Condino F, et al. MR imaging of middle cerebellar peduncle width: differentiation of multiple system atrophy from Parkinson disease. Radiology 2006;239:825-830.

26. Litvan I, Agid Y, Calne D, et al. Clinical research criteria for the diagnosis of progressive supranuclear palsy (Steele-Richardson-Olszewski syndrome): report of the NINDS-SPSP international workshop. Neurology 1996;47:1-9.

27. Oba H, Yagishita A, Terada H, et al. New and reliable MRI diagnosis for progressive supranuclear palsy. Neurology 2005;64:2050-2055.

28. Gröschel K, Kastrup A, Litvan I, Schulz JB. Penguins and hummingbirds: midbrain atrophy in progressive supranuclear palsy. Neurology 2006;66:949-950.

29. Wszolek ZK, Slowinski J, Imamura A, Tsuboi Y, Broderick DF. New and reliable MRI diagnosis for progressive supranuclear palsy. Neurology 2006;66:781.

30. Borroni B, Malinverno M, Gardoni F, et al. A combination of CSF tau ratio and midsaggittal midbrain-to-pons area ratio atrophy for the early diagnosis of progressive supranuclear palsy. J Alzheimers Dis 2010;22:195-203.

31. Arabia G, Quattrone A. The midbrain to pons ratio: a simple and specific MRI sign of progressive supranuclear palsy. Neurology 2013;81:2147.

32. Massey LA, Jäger HR, Paviour DC, et al. The midbrain to pons ratio: a simple and specific MRI sign of progressive supranuclear palsy. Neurology 2013;80:1856-1861.

33. Morelli M, Arabia G, Messina D, et al. Effect of aging on magnetic resonance measures differentiating progressive supranuclear palsy from Parkinson's disease. Mov Disord 2014;29:488-495.

34. Litvan I, Bhatia KP, Burn DJ, et al. Movement disorders society scientific issues committee report: SIC task force appraisal of clinical diagnostic criteria for Parkinsonian disorders. Mov Disord 2003;18:467-486.

\section{Save These Dates for AAN CME Opportunities!}

Mark these dates on your calendar for exciting continuing education conferences by the American Academy of Neurology. Learn more at AAN.com/conferences.

\section{Fall Conference}

- October 14-16, 2016, Las Vegas, NV, at the Cosmopolitan of Las Vegas

\section{Breakthroughs in Neurology}

- January 13-16, 2017, Phoenix, AZ, at the Sheraton Grand at Wild Horse Pass

\section{AAN Annual Meeting}

- April 22-28, 2017, Boston, MA, at the Boston Convention Center 


\section{Neurology}

\section{MR parkinsonism index predicts vertical supranuclear gaze palsy in patients with PSP- parkinsonism}

Aldo Quattrone, Maurizio Morelli, David R. Williams, et al.

Neurology 2016;87;1266-1273 Published Online before print August 24, 2016

DOI 10.1212/WNL.0000000000003125

This information is current as of August 24, 2016

\section{Updated Information \&} Services

References

Subspecialty Collections

Permissions \& Licensing

Reprints including high resolution figures, can be found at: http://n.neurology.org/content/87/12/1266.full

This article cites 33 articles, 11 of which you can access for free at: http://n.neurology.org/content/87/12/1266.full\#ref-list-1

This article, along with others on similar topics, appears in the following collection(s):

MRI

http://n.neurology.org/cgi/collection/mri

Parkinson's disease/Parkinsonism

http://n.neurology.org/cgi/collection/parkinsons_disease_parkinsonism Progressive supranuclear palsy

http://n.neurology.org/cgi/collection/progressive_supranuclear_palsy

Information about reproducing this article in parts (figures,tables) or in its entirety can be found online at:

http://www.neurology.org/about/about_the_journal\#permissions

Information about ordering reprints can be found online:

http://n.neurology.org/subscribers/advertise

Neurology ${ }^{\circledR}$ is the official journal of the American Academy of Neurology. Published continuously since 1951, it is now a weekly with 48 issues per year. Copyright () 2016 American Academy of Neurology. All rights reserved. Print ISSN: 0028-3878. Online ISSN: 1526-632X.

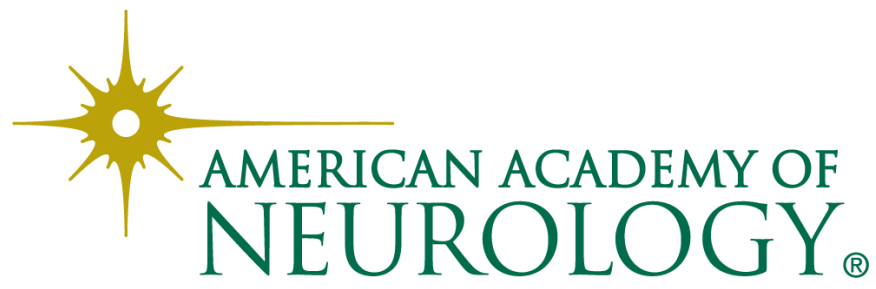

